\title{
Role of Protein and Hydrolysates Before Exercise
}

\begin{abstract}
Kevin D. Tipton
Adaptations to exercise training are determined by the response of metabolic and molecular mechanisms that determine changes in proteins. The type, intensity, and duration of exercise, as well as nutrition, determine these responses. The importance of protein, in the form of intact proteins, hydrolysates, or free amino acids, for exercise adaptations is widely recognized. Exercise along with protein intake results in accumulation of proteins that influence training adaptations. The total amount of protein necessary to optimize adaptations is less important than the type of protein, timing of protein intake, and the other nutrients ingested concurrently with the protein. Acute metabolic studies offer an important tool to study the responses of protein balance to various exercise and nutritional interventions. Recent studies suggest that ingestion of free amino acids plus carbohydrates before exercise results in a superior anabolic response to exercise than if ingested after exercise. However, the difference between pre- and postexercise ingestion of intact proteins is not apparent. Thus, the anabolic response to exercise plus protein ingestion seems to be determined by the interaction of timing of nutrient intake in relation to exercise and the nutrients ingested. More research is necessary to delineate the optimal combination of nutrients and timing for various types of training adaptations. Protein and amino acid intake have long been deemed important for athletes and exercising individuals. Olympic athletes, from the legendary Milo to many in the 1936 Berlin games, reportedly consumed large amounts of protein. Modern athletes may consume slightly less than these historical figures, yet protein is deemed extremely important by most. Protein is important as a source of amino acids for recovery from exercise and repair of damaged tissues, as well as for adaptations to exercise training, such as muscle hypertrophy and mitochondrial biogenesis.
\end{abstract}

Key Words: net muscle protein synthesis, essential amino acids, resistance exercise, muscle hypertrophy

Clearly, protein intake is important for athletes and others to support exercise and training. However, the amount of dietary protein to optimize exercise performance and adaptation to training is difficult to determine. A high protein intake is often thought to be critical for muscle growth and repair and enhancement of training adaptations, and a huge supplement industry has been built on this assumption.

The author is with the Exercise Metabolism Research Group, School of Sport and Exercise Sciences, University of Birmingham, Birmingham B29 5SA UK. 
Furthermore, protein is utilized for fuel during exercise, albeit to a relatively limited extent, and is important for mitochondrial biogenesis, hormone production, immune function, and many other physiological processes. The scientific evidence for the efficacy of high protein intakes for exercisers is, at best, equivocal, and has been extensively debated in the scientific community $(21,27,28,31,39,40,54,55)$. A wide-ranging discussion of this issue is beyond the scope of this article but is covered in detail by Phillips in another review in this supplemental issue (33).

There seem to be 2 main reasons for the controversy over protein intake for athletes and exercisers. First, the methods used to determine protein requirements, for example, $\mathrm{N}$ balance and amino acid oxidation, are not particularly relevant to athletes (54). Perhaps more important, the recommendation of an exact figure, or even a range, is predicated on the assumption that there is a linear relationship between the amount of protein ingested and the resulting desirable adaptation of the muscle. It is now clear that the uptake of amino acids from ingested protein is variable and dependent on many factors other than simply the amount ingested. The type of protein $(49,57)$ or amino acids $(9,49,51)$, concurrently ingested nutrients $(9,15,26)$, and the timing of intake in relation to exercise $(48,52)$ all influence the uptake of amino acids from protein sources. Thus, a particular recommendation for protein intake would seem somewhat nonsensical. The focus of this review is on the timing of protein and amino acid sources in relation to exercise with particular emphasis on the response of muscle to ingestion of amino acid sources before exercise.

\section{Metabolic Response to Exercise}

Adaptations to exercise training are specific to the type of exercise performed. In general, it can be said that resistance training results in increased muscle size and strength, particularly in Type II fibers. Endurance training results in increased oxidative capacity largely via increased number and size of mitochondria. These adaptations result from changes in the amount of various proteins in response to exercise. It is becoming increasingly clear that adaptations to training result from the accumulation of proteins caused by transient metabolic and molecular responses to individual exercise bouts $(16,17)$. The responses are influenced by the type, intensity, and duration of exercise and nutrient intake. Changes in protein levels result from the responses of both muscle protein synthesis and breakdown to exercise $(53,55)$.

The metabolic mechanism for changes in protein levels is the balance between the rates of synthesis and breakdown. Increased synthesis in relation to breakdown will result in positive protein balance and gains in protein. If breakdown is greater than synthesis, proteins will be lost. The changes in synthesis and breakdown of each individual protein must be controlled to account for changes that manifest themselves as phenotypic adaptation. It is possible to measure whole-body and tissue rates of protein synthesis, as well as synthesis rates of types of proteins (42) or even some individual proteins $(2,3,42)$. Measurement of breakdown rates for individual proteins has yet to be perfected (38), however, and thus we are limited primarily to whole-body and tissue measurements of mixed-protein breakdown rates. Thus, our knowledge of the response of protein breakdown to exercise and nutrition is somewhat limited relative to what is known about the protein synthetic response. 
However, the response of net muscle protein balance to exercise and nutrition results primarily from changes in synthesis rather than breakdown $(5,51)$.

The primary response of muscle protein metabolism to exercise occurs after rather than during the event. During exercise muscle protein synthesis may be decreased (13) or unchanged from resting levels (14). After exercise, muscle protein synthesis and breakdown are increased for as much as $24-48 \mathrm{~h}(4,35)$. The increase in protein synthesis is greater than the increase in breakdown, resulting in increased net muscle protein balance $(4,35)$. Without ingestion of nutrients, particularly a source of amino acids, net muscle protein balance does not reach positive, that is, anabolic, levels $(4,35,51)$. There has been a great deal of attention given to ingestion of amino acids and other nutrients after exercise but only minimal investigation of the response of muscle to nutrient ingestion before exercise. A comprehensive investigation of the topic is beyond the scope of this review; thus the reader is referred to any of the many recent reviews that are relevant $(31,32,40,53,54,55$, $58,59)$, including the others in this supplemental issue.

\section{Methodology}

The impact of timing of protein intake in relation to exercise on muscle protein metabolism has begun to receive more attention over the last few years $(24,37$, $43,44,48,52)$. Several studies have used a longitudinal approach to address the importance of timing of protein intake. Ultimately, the importance of protein and amino acid nutrition for exercising individuals comes down to the adaptations that result. Thus, measurement of changes in muscle size, strength, or oxidative capacity should be the bottom line. Unfortunately, the differences between different nutrient-intake regimens are usually quite small, albeit possibly very important. The expense and difficulty in controlling (e.g., dietary intake, sleep, emotional issues, training) these studies means that most are limited to periods of weeks to months with relatively small sample sizes. Therefore, one potential problem with longitudinal endpoint studies is that the results may not be representative of the actual physiological situation (50). Many of these studies fail to show a difference between treatments; lack of measurable difference does not necessarily indicate that no difference exists. Failure to detect a difference between treatments may mean merely that a real difference between groups was not detected because of lack of sufficient control, small number of subjects, imprecise measurement of endpoints, or the inability to carry out the study for a sufficient period of time. As a result of these difficulties, interpreting the results is often difficult, and conclusions may be rather equivocal both within and between studies.

Over the past few years, we have attempted to delineate the metabolic response of muscle to nutrient intake and exercise, particularly resistance exercise (for reviews see Tipton et al. $[53,54,55])$. These studies investigated metabolic control of muscle protein metabolism. Moreover, if the assumption is made that the acute metabolic response represents the potential for long-term changes, results may be interpreted to discern phenotypic adaptations likely given various nutrient interventions (50). This assumption was investigated in several recent studies $(30,47,50,54)$. The results of those studies indicate that the measurement of the acute metabolic response to exercise and nutrient intake provides important information that may be used to delineate the long-term response of changes in muscle protein. 
More recently, we conducted a series of studies aimed at examining the impact on the anabolic response to exercise of timing of nutrient intake both before and after exercise $(37,48,52)$. The primary endpoint for these studies is the area under the curve of amino acid uptake. Blood samples were taken from the femoral artery and vein before and after ingestion of nutrients along with an intense resistanceexercise routine. Amino acid concentrations were measured in the blood samples, and blood flow was measured using indocyanine green dye. Blood samples were taken periodically, and the net balance of the amino acids was calculated by multiplying the arteriovenous difference by the blood flow. The net amino acid balance was taken to represent the net muscle protein balance, and the area under the curve of the net balance represents the response of the muscle over the time of measurement-generally $3-4 \mathrm{~h}$ in these studies $(37,48,52)$. Positive values for area under the curve represent net uptake of amino acids, and negative values, net release. Furthermore, delivery of amino acids to the muscle was determined by multiplying the arterial amino acid concentration by the blood flow.

There are several assumptions that must be met for these measurements to represent the anabolic response of the muscle. First, uptake of the amino acids represents incorporation into muscle protein. The amino acid chosen for measurement must not be oxidized in the muscle, and it must be an essential amino acid. Thus, the branched-chain amino acids or nonessential amino acids such as alanine and glycine are not suitable for these arteriovenous balance studies. Leucine oxidation is increased by both exercise (25) and leucine intake (60), so incorporation of leucine into protein cannot be distinguished from leucine oxidation when leucine is taken up by the muscle. Essential amino acids, such as phenylalanine and threonine, which are not oxidized or produced in muscle (56), are suitable to represent uptake and incorporation into muscle protein in arteriovenous balance studies.

Another issue to consider is the potential expansion of the free amino acid pool in muscle. If the size of the free amino acid pool increases as a result of amino acid uptake only to be subsequently released before the end of the measurement period, the measured uptake does not represent net muscle protein synthesis. It is clear that increased arterial amino acid amino acid after amino acid or protein intake results in expansion of the intracellular free pool with subsequent release of the amino acids back into the venous blood without incorporation of some of the amino acids (47). Thus, these studies must account for the amino acids that are taken up but not incorporated by making the period of measurement long enough to include the release of the amino acids, measuring the intracellular free pool and subtracting the amino acids remaining, or both. In our studies, muscle biopsies were taken before the ingestion of the investigated nutrient solution and at the end of the measurement period $(10,47,48,49,52)$. The intracellular amino acid concentration was calculated and subtracted from the calculated uptake by the muscle. The resulting value then gives the net muscle protein synthesis, assuming that all amino acids remaining in the pool will be released, that is, the lower end of the possible range of net muscle protein synthesis $(10,18,47,48,49,52)$. Therefore, it is possible to determine the higher and lower ends of the range of possible values for net muscle protein synthesis.

Finally, we may determine the uptake of ingested amino acids by calculating the amino acid uptake divided by the amount ingested. This value provides an indication of response of net muscle protein synthesis relative to the amount of 
the measured amino acid in the ingested protein. Uptake of a particular amino acid will increase along with increasing amounts ingested, thus potentially causing an over- or underestimate of the amount of net muscle protein synthesis as a result of protein ingestion $(18,49)$. Given these considerations, arteriovenous balance of single amino acids may be used to determine differences in the response of muscle anabolism to various exercise and nutrient interventions, such as differences in timing of nutrient intake $(37,48,52)$.

\section{Timing of Ingestion of Amino Acid Sources in Relation to Exercise}

Postexercise nutrient ingestion has been investigated by several previous researchers $(24,37,44)$, but only recently has the anabolic response of muscle to preexercise feeding been examined and compared with postexercise responses. In an earlier investigation, untrained volunteers ingested $6 \mathrm{~g}$ of essential amino acids plus $35 \mathrm{~g}$ of carbohydrate immediately before initiation and immediately after completion of an intense, leg-resistance-exercise routine (52). Net muscle protein balance was determined, and expansion of the free amino acid pool was accounted for by measuring intracellular amino concentrations from muscle biopsies before nutrient ingestion and at the end of the measurement period. We found that amino acid uptake was greater when the nutrients were ingested before the exercise bout than immediately afterward (52). Furthermore, when compared with a previous investigation using identical methods (37), the anabolic response to amino acid/ carbohydrate ingestion before exercise is superior to that after exercise. Finally, the immediate anabolic response to ingestion of these nutrients is similar whether they are ingested at 1 or $3 \mathrm{~h}$ postexercise (37). Thus, whereas a comparison based on the same measurements, that is, amino acid uptake over $3 \mathrm{~h}$ of measurement, cannot be made for the 3-h-postexercise time point, it is at least intuitively obvious that the anabolic response to exercise and amino acid/carbohydrate ingestion is greater with preexercise ingestion versus immediately, 1 , and $3 \mathrm{~h}$ postexercise. In fact, when compared with the response to many different types and combinations of nutrients and timing of ingestion, the utilization of amino acids from ingested proteins or amino acids is greatest when essential amino acids combined with carbohydrates are consumed before exercise (54).

It is important to emphasize that the amino acid source that provided the greatest response was in free form and was combined with carbohydrates (52). Because ingestion of free amino acids for most people is less likely-because of taste, cost, and other issues-we decided to examine the response of muscle protein metabolism to whey proteins before exercise (48). Untrained volunteers ingested $20 \mathrm{~g}$ of whey proteins either immediately before resistance exercise or 1 $\mathrm{h}$ postexercise. We chose $1 \mathrm{~h}$ postexercise because, based on our previous studies, the 1-h response was greater than immediately postexercise $(52,37)$. There was little, if any, difference in amino acid utilization between the pre- and postexercise treatments. Thus, it seems that, in respect to the response of muscle anabolism to timing of ingestion, not all amino acid sources are alike. The response is variable depending on not only the timing of amino acid intake but also the combination of timing and source of amino acids or perhaps the concurrently ingested nutrients. 
The reasons for the difference in response between essential amino acids and carbohydrates and whey proteins are not immediately obvious. There are several differences between the 2 studies that may contribute to the differences between them. There was no carbohydrate ingested along with the whey protein (48). It is unlikely that the differences in insulin response would explain the different responses of amino acid uptake after ingestion of essential amino acids or protein because the insulin responses were similar $(48,52)$. The postexercise timing of ingestion was different between studies. Amino acids and carbohydrates were compared immediately before exercise with immediately after exercise, but whey-protein ingestion was compared immediately before exercise with $1 \mathrm{~h}$ after exercise (48). We chose $1 \mathrm{~h}$ because the response to ingestion of essential amino acids at $1 \mathrm{~h}$ after exercise (37) was slightly greater than immediately after exercise (52). However, we cannot rule out the possibility that amino acid uptake from whey proteins would not be the same as free amino acids ingested at different times after exercise. Furthermore, more total energy was ingested in the free amino acid plus carbohydrate treatment. A total of $164 \mathrm{kcal}$ were ingested (26) versus $80 \mathrm{kcal}$ during the whey-protein treatment (48). Whereas indications are that the anabolic response to acute ingestion depends more on the amino acids ingested than on the total energy intake $(9,24$, $26,44)$, the influence of energy cannot be ruled out.

The most likely reason that free amino acids ingested before exercise lead to superior amino acid uptake versus after exercise is differences in delivery of amino acids to the muscle. Amino acid delivery, particularly during exercise, was not different between pre- and postexercise ingestion of whey proteins (48), but preexercise ingestion of free amino acids resulted in much greater amino acid delivery during exercise than postexercise ingestion (52). Thus, if delivery of amino acids to the leg is a key component for the anabolic response (5), these data suggest that it is delivery that explains the differences between whey proteins and free amino acids.

These studies provide no specific data to allow a clear explanation of why amino acid delivery was greater for free amino acids ingested before than after exercise but not different for whey proteins. Digestion of the protein may limit the amount of amino acids in the blood during exercise when blood flow is high, thus reducing delivery compared with free amino acids. Clearly, digestive properties of proteins influence the anabolic response at rest (11) and after exercise (57). Support for this notion is provided by inspection of the amino acid concentration and delivery data during exercise. The increase in arterial amino acid concentrations during exercise was $\sim 3$-fold greater for essential amino acids (52) than for whey proteins (48) when ingested immediately before exercise. Thus, the delivery of phenylalanine during exercise was increased $\sim 70 \%$ more after ingestion of essential amino acids (52) than whey proteins (48). Given more time for digestion of the proteins before exercise, it is possible that delivery of amino acids may approach levels similar to those of free amino acids. Thus, consuming intact proteins at other time points before exercise, for example, 15, 30, 45 or $60 \mathrm{~min}$, may result in a greater anabolic response than immediately before exercise. Other forms for ingestion of amino acids may be considered. At present there are no studies examining the response of muscle protein anabolism to ingestion of protein hydrolysates before exercise. Early reports suggested that protein hydrolysates may be absorbed more readily than free amino acids $(19,45)$; however, increased absorption of amino acids does not seem 
to lead to superior nutritional quality $(29,41)$. Efficacy of protein hydrolysates has been tested primarily in clinical settings $(29,41)$, so the applicability of these data to an exercise situation is questionable, at best. To our knowledge, no data from measurements of peptide absorption or the impact on protein metabolism exist in humans in relation to exercise.

There is one other possibility that may help explain the differences in the anabolic response to timing of ingestion of essential amino acids and whey proteins. Amino acids serve not only to supply substrate for synthesis of muscle proteins but also as signaling molecules. A detailed discussion of the influence of amino acids on muscle-signaling pathways is beyond the scope of this review. The reader is referred to any of the many excellent reviews on this topic $(8,20,21,53)$, including an accompanying article in this supplemental issue (22). Amino acids, particularly leucine (54), stimulate translation-initiation pathways, resulting in increased muscle protein synthesis - both at rest $(6)$ and after exercise $(6,51)$. During exercise, AMPK activity is stimulated, resulting in an inhibition of translation initiation $(7,23)$. When amino acids are ingested before exercise, translation-initiation pathways may be stimulated (22), resulting in increased muscle protein synthesis during exercise (52). Whereas the response of translation initiation to preexercise ingestion of an amino acid source would result in increased muscle protein synthesis and thus amino acid uptake during exercise, it is unclear how this response may explain the differences between essential amino acids and whey proteins. Both sources of amino acids should stimulate the initiation pathways. At this time, however, there are no data to suggest any reasons for differences between the 2 sources.

Clearly, there is a great deal of information yet to be determined before definitive conclusions can be made concerning the optimal timing of nutrient ingestion in relation to exercise. Undoubtedly, the basis of the response is multifactorial. There are many factors that may influence the anabolic response, including sex (46), age (12), and training status $(34,36)$ of the individual; type of protein (49, $57)$ or form of amino acids $(26,48,52)$ ingested; and other nutrients concurrently ingested $(9,15,26,52)$. The interaction of any or all of these factors indisputably plays a role, as well. Therefore, further examination of the response to ingestion of intact proteins, as well as protein hydrolysates, alone and in combination with other nutrients at various time points before exercise may be warranted to determine the optimal time for ingestion for muscle protein anabolism.

\section{References}

1. Anthony, J.C., T.G. Anthony, S.R. Kimball, and L.S. Jefferson. Signaling pathways involved in translational control of protein synthesis in skeletal muscle by leucine. $J$. Nutr. 131:856S-860S, 2001.

2. Balagopal, P., O. Ljungqvist, and K.S. Nair. Skeletal muscle myosin heavy-chain synthesis rate in healthy humans. Am. J. Physiol. 272:E45-E50, 1997.

3. Balagopal, P., K.S. Nair, and W.S. Stirewalt. Isolation of myosin heavy chain from small skeletal muscle samples by preparative continuous elution gel electrophoresis: application to measurement of synthesis rate in human and animal tissue. Anal. Biochem. 221:72-77, 1994.

4. Biolo, G., S.P. Maggi, B.D. Williams, K.D. Tipton, and R.R. Wolfe. Increased rates of muscle protein turnover and amino acid transport after resistance exercise in humans. Am. J. Physiol. 268:E514-E520, 1995. 
5. Biolo, G., K.D. Tipton, S. Klein, and R.R. Wolfe. An abundant supply of amino acids enhances the metabolic effect of exercise on muscle protein. Am. J. Physiol. 273:E122E129, 1997.

6. Biolo, G., K.D. Tipton, S. Klein, and R.R. Wolfe. Enhanced protein synthesis in skeletal muscle after resistance exercise during hyperaminoacidemia: role of blood flow and amino acid transport. Am. J. Physiol. Endocrinol. Metab. 273:E122-E129, 1997.

7. Bolster, D.R., S.J. Crozier, S.R. Kimball, and L.S. Jefferson. AMP-activated protein kinase suppresses protein synthesis in rat skeletal muscle through down-regulated mammalian target of rapamycin (mTOR) signaling. J. Biol. Chem. 277:23977-23980, 2002.

8. Bolster, D.R., L.S. Jefferson, and S.R. Kimball. Regulation of protein synthesis associated with skeletal muscle hypertrophy by insulin-, amino acid- and exercise-induced signalling. Proc. Nutr. Soc. 63:351-356, 2004.

9. Borsheim, E., A. Aarsland, and R.R. Wolfe. Effect of an amino acid, protein, and carbohydrate mixture on net muscle protein balance after resistance exercise. Int. J. Sport Nutr. Exerc. Metab. 14:255-271, 2004.

10. Borsheim, E., K.D. Tipton, S.E. Wolf, and R.R. Wolfe. Essential amino acids and muscle protein recovery from resistance exercise. Am. J. Physiol. Endocrinol. Metab. 283:E648-E657, 2002.

11. Dangin, M., Y. Boirie, C. Guillet, and B. Beaufrere. Influence of the protein digestion rate on protein turnover in young and elderly subjects. J. Nutr. 132:3228S-3233S, 2002.

12. Dangin, M., C. Guillet, C. Garcia-Rodenas, et al. The rate of protein digestion affects protein gain differently during aging in humans. J. Physiol. 549:635-644, 2003.

13. Dreyer, H.C., S. Fujita, J.G. Cadenas, D.L. Chinkes, E. Volpi, and B.B. Rasmussen. Resistance exercise increases AMPK activity and reduces 4E-BP1 phosphorylation and protein synthesis in human skeletal muscle. J Physiol. 576:613-624, 2006.

14. Durham, W.J., S.L. Miller, C.W. Yeckel, et al. Leg glucose and protein metabolism during an acute bout of resistance exercise in humans. J. Appl. Physiol. 97:1379-1386, 2004.

15. Elliot, T.A., M.G. Cree, A.P. Sanford, R.R. Wolfe, and K.D. Tipton. Milk ingestion stimulates net muscle protein synthesis following resistance exercise. Med. Sci. Sports Exerc. 38:667-674, 2006.

16. Hawley, J.A., M. Hargreaves, and J.R. Zierath. Signalling mechanisms in skeletal muscle: role in substrate selection and muscle adaptation. Essays Biochem. 42:1-12, 2006.

17. Hawley, J.A., K.D. Tipton, and M.L. Millard-Stafford. Promoting training adaptations through nutritional interventions. J. Sports Sci. 24:1-13, 2006.

18. Katsanos, C.S., D.L. Chinkes, M. Sheffield-Moore, A. Aarsland, H. Kobayashi, and R.R. Wolfe. Method for the determination of the arteriovenous muscle protein balance during non-steady-state blood and muscle amino acid concentrations. Am. J. Physiol. Endocrinol. Metab. 289:E1064-E1070, 2005.

19. Keohane, P.P., G.K. Grimble, B. Brown, R.C. Spiller, and D.B. Silk. Influence of protein composition and hydrolysis method on intestinal absorption of protein in man. Gut. 26:907-913, 1985.

20. Kimball, S.R., P.A. Farrell, and L.S. Jefferson. Invited review: role of insulin in translational control of protein synthesis in skeletal muscle by amino acids or exercise. $J$. Appl. Physiol. 93:1168-1180, 2002.

21. Kimball, S.R., and L.S. Jefferson. Signaling pathways and molecular mechanisms through which branched-chain amino acids mediate translational control of protein synthesis. J. Nutr. 136:227S-231S, 2006.

22. Koopman, R. Role of amino acids and peptides in the molecular signaling in skeletal muscle after resistance. Int. J. Sport Nutr. Exerc. Metab. 17(Suppl.):S47-S57, 2007.

23. Koopman, R., A.H. Zorenc, R.J. Gransier, D. Cameron-Smith, and L.J. van Loon. Increase in S6K1 phosphorylation in human skeletal muscle following resistance 
exercise occurs mainly in Type II muscle fibers. Am. J. Physiol. Endocrinol. Metab. 290:E1245-E1252, 2006.

24. Levenhagen, D.K., J.D. Gresham, M.G. Carlson, D.J. Maron, M.J. Borel, and P.J. Flakoll. Postexercise nutrient intake timing in humans is critical to recovery of leg glucose and protein homeostasis. Am. J. Physiol. Endocrinol. Metab. 280:E982-E993, 2001.

25. McKenzie, S., S.M. Phillips, S.L. Carter, S. Lowther, M.J. Gibala, and M.A. Tarnopolsky. Endurance exercise training attenuates leucine oxidation and BCOAD activation during exercise in humans. Am. J. Physiol. Endocrinol. Metab. 278:E580-E587, 2000.

26. Miller, S.L., K.D. Tipton, D.L. Chinkes, S.E. Wolf, and R.R. Wolfe. Independent and combined effects of amino acids and glucose after resistance exercise. Med. Sci. Sports Exerc. 35:449-455, 2003.

27. Millward, D.J. Protein and amino acid requirements of athletes. J. Sports Sci. 22:143144, 2004.

28. Millward, D.J., J.L. Bowtell, P. Pacy, and M.J. Rennie. Physical activity, protein metabolism and protein requirements. Proc. Nutr. Soc. 53:223-240, 1994.

29. Moriarty, K.J., J.E. Hegarty, P.D. Fairclough, M.J. Kelly, M.L. Clark, and A.M. Dawson. Relative nutritional value of whole protein, hydrolysed protein and free amino acids in man. Gut. 26:694-699, 1985.

30. Paddon-Jones, D., M. Sheffield-Moore, R.J. Urban, et al. Essential amino acid and carbohydrate supplementation ameliorates muscle protein loss in humans during 28 days bedrest. J. Clin. Endocrinol. Metab. 89:4351-4358, 2004.

31. Phillips, S.M. Protein requirements and supplementation in strength sports. Nutrition. 20:689-695, 2004.

32. Phillips, S.M. Dietary protein for athletes: from requirements to metabolic advantage. Appl. Physiol. Nutr. Metab. In Press.

33. Phillips, S.M., D.R. Moore, and J.E. Tang. A critical examination of dietary protein requirements, benefits, and excesses in athletes. Int. J. Sport Nutr. Exerc. Metab. 17(Suppl.):S58-S76, 2007.

34. Phillips, S.M., G. Parise, B.D. Roy, K.D. Tipton, R.R. Wolfe, and M.A. Tamopolsky. Resistance-training-induced adaptations in skeletal muscle protein turnover in the fed state. Can. J. Physiol. Pharmacol. 80:1045-1053, 2002.

35. Phillips, S.M., K.D. Tipton, A. Aarsland, S.E. Wolf, and R.R. Wolfe. Mixed muscle protein synthesis and breakdown following resistance exercise in humans. Am. J. Physiol. Endocrinol. Metab. 273:E99-E107, 1997.

36. Phillips, S.M., K.D. Tipton, A.A. Ferrando, and R.R. Wolfe. Resistance training reduces the acute exercise-induced increase in muscle protein turnover. Am. J. Physiol. 276: E118-E124, 1999.

37. Rasmussen, B.B., K.D. Tipton, S.L. Miller, S.E. Wolf, and R.R. Wolfe. An oral essential amino acid-carbohydrate supplement enhances muscle protein anabolism after resistance exercise. J. Appl. Physiol. 88:386-392, 2000.

38. Rennie, M.J., and D.J. Millward. 3-Methylhistidine excretion and the urinary 3-methylhistidine/creatinine ratio are poor indicators of skeletal muscle protein breakdown. Clin. Sci. (Lond.). 65:217-225, 1983.

39. Rennie, M.J., and K.D. Tipton. Protein and amino acid metabolism during and after exercise and the effects of nutrition. Annu. Rev. Nutr. 20:457-483, 2000.

40. Rennie, M.J., H. Wackerhage, E.E. Spangenburg, and F.W. Booth. Control of the size of the human muscle mass. Annu. Rev. Physiol. 66:799-828, 2004.

41. Rigo, J., B.L. Salle, J.C. Picaud, G. Putet, and J. Senterre. Nutritional evaluation of protein hydrolysate formulas. Eur. J. Clin. Nutr. 49(Suppl. 1):S26-S38, 1995.

42. Rooyackers, O.E., P. Balagopal, and K.S. Nair. Measurement of synthesis rates of specific muscle proteins using needle biopsy samples. Muscle Nerve. 5(Suppl.):S93-S96, 1997.

43. Roy, B.D., K. Luttmer, M.J. Bosman, and M.A. Tarnopolsky. The influence of postexercise macronutrient intake on energy balance and protein metabolism in active 
females participating in endurance training. Int. J. Sport Nutr. Exerc. Metab. 12:172188, 2002.

44. Roy, B.D., M.A. Tarnopolsky, J.D. MacDougall, J. Fowles, and K.E. Yarasheski. Effect of glucose supplement timing on protein metabolism after resistance training. J. Appl. Physiol. 82:1882-1888, 1997.

45. Silk, D.B., G.K. Grimble, and R.G. Rees. Protein digestion and amino acid and peptide absorption. Proc. Nutr. Soc. 44:63-72, 1985.

46. Tipton, K.D. Gender differences in protein metabolism. Curr. Opin. Clin. Nutr. Metab. Care. 4:493-498, 2001.

47. Tipton, K.D., E. Borsheim, S.E. Wolf, A.P. Sanford, and R.R. Wolfe. Acute response of net muscle protein balance reflects $24-\mathrm{h}$ balance after exercise and amino acid ingestion. Am. J. Physiol. Endocrinol. Metab. 284:E76-E89, 2003.

48. Tipton, K.D., T.A. Elliott, M.G. Cree, A.A. Aarsland, A.P. Sanford, and R.R. Wolfe. Stimulation of net muscle protein synthesis by whey protein ingestion before and after exercise. Am. J. Physiol. Endocrinol. Metab. 292:E71-E76, 2007.

49. Tipton, K.D., T.A. Elliott, M.G. Cree, S.E. Wolf, A.P. Sanford, and R.R. Wolfe. Ingestion of casein and whey proteins result in muscle anabolism after resistance exercise. Med. Sci. Sports Exerc. 36:2073-2081, 2004.

50. Tipton, K.D., and A.A. Ferrando. Improving muscle mass: response of muscle metabolism to exercise, nutrition and anabolic agents. In: Drugs and Ergogenic Aids to Improve Sport Performance (Essays in Biochemistry, Vol. 43), C. Cooper and J. Shearman (Eds.). London: Portland Press. In press.

51. Tipton, K.D., A.A. Ferrando, S.M. Phillips, D. Doyle, Jr., and R.R. Wolfe. Postexercise net protein synthesis in human muscle from orally administered amino acids. Am. J. Physiol. 276:E628-E634, 1999.

52. Tipton, K.D., B.B. Rasmussen, S.L. Miller, et al. Timing of amino acid-carbohydrate ingestion alters anabolic response of muscle to resistance exercise. Am. J. Physiol. Endocrinol. Metab. 281:E197-E206, 2001.

53. Tipton, K.D., and C.P. Sharp. The response of intracellular signaling and muscle protein metabolism to nutrition and exercise. Eur. J. Sports Sci. 5:107-121, 2005.

54. Tipton, K.D., and O.C. Witard. Protein requirements and recommendations for athletes: relevance of ivory tower arguments for practical recommendations. Clin. Sports Med. 26:17-36, 2007.

55. Tipton, K.D., and R.R. Wolfe. Protein and amino acids for athletes. J Sports Sci. 22:6579, 2004.

56. Tourian, A., J. Goddard, and T.T. Puck. Phenylalanine hydroxylase activity in mammalian cells. J. Cell Physiol. 73:159-170, 1969.

57. Wilkinson, S.B., M.A. Tarnopolsky, M.J. Macdonald, J.R. MacDonald, D. Armstrong, and S.M. Phillips. Consumption of fluid skim milk promotes greater muscle protein accretion after resistance exercise than does consumption of an isonitrogenous and isoenergetic soy-protein beverage. Am. J. Clin. Nutr. 85:1031-1040, 2007.

58. Wolfe, R.R. Effects of amino acid intake on anabolic processes. Can. J. Appl. Physiol. 26(Suppl.):S220-S227, 2001.

59. Wolfe, R.R. Regulation of muscle protein by amino acids. J. Nutr. 132:3219S-3224S, 2002.

60. Young, V.R., A.E. El Khoury, C.A. Raguso, A.H. Forslund, and L. Hambraeus. Rates of urea production and hydrolysis and leucine oxidation change linearly over widely varying protein intakes in healthy adults. J. Nutr. 130:761-766, 2000. 